\title{
Mechanism of Spine Motion About Contact Time in Quadruped Running
}

\author{
Qi Liu', Yannian Bao ${ }^{1}$, Wei Yu' ${ }^{1}$, Jianming Zhang ${ }^{1}$, Chao Li² and Xinru Xie²
}

\begin{abstract}
The current research of quadruped robot focuses on the quadruped robot with spine motion. Contact time is a very important part of system performance. However, the mechanism of spine motion about contact time has not been clearly elucidated. In this paper, the effect of spine motion on contact time is studied deeply from dynamic view. Firstly, a simplified model of the quadruped robot with spine joint is set up, its dynamic equations are derivated, and a method that can generate passive periodic locomotion is proposed. Secondly, according to the vertical spring oscillator model, the two-dimension planar locomotion of the simplified model is regarded as a special vibration in the vertical direction, and the approximate formula of calculating contact time is obtained. Finally, the approximate formula of calculating contact time is verified by the simulation results of passive periodic locomotion, and the effect of spine motion on contact time is deeply discussed based on the approximate formula of calculating contact time. The discussion proves that spine motion indeed has little effect on contact time, but spine motion can slightly reduce body pith movement and regulate the leg stiffness in leg contact phase. This research proposes an effective research method which can be used to study the motion mechanism of the quadruped robot with spine motion, and the mechanism of spine motion about contact time is clearly elucidated which is helpful to set the parameters of mechanical structure and study control algorithm about the quadruped robot with spine motion.
\end{abstract}

Keywords: Quadruped robot, Spine motion, Passive locomotion, Contact time, Leg stiffness

\section{Introduction}

Developing a quadruped robot that has ascendant locomotion performance like quadruped mammals is always the dream of researchers. The research about quadruped robot was originated from the 60s of last century when the walking quadruped robot was the focus of study. The most typical one is the "Walking Truck" made by Mosher [1]. From the 1980s, researchers started to research the dynamic quadruped robot. When working in MIT lab, Raibert [2] clearly expounded the balance of the dynamic quadruped robot from dynamic view. Afterwards, Raibert et al. [3] developed a dynamic quadruped robot for the U.S. Army on the basis of his theory. This dynamic quadruped robot was called BigDog and its locomotion performance was amazing. After Raibert's study, many

\footnotetext{
*Correspondence: lq6688@caep.cn

${ }^{1}$ Institute of Chemical Materials, China Academy of Engineering Physics, Mianyang 621999, China

Full list of author information is available at the end of the article
}

aspects of the dynamic quadruped robot are studied deeply. Spröwitz et al. [4] researched the self-stabilizing behavior of the dynamic quadruped robot. Miller et al. [5] studied the measure for quantifying disturbance rejection of dynamic running systems in an unstructured environment. Ding et al. [6] researched the foot-terrain interaction mechanics for legged robots through model and experiment analysis. Kalakrishnan et al. [7] presented an effective control architecture for fast quadruped locomotion over rough terrain. In the 2010s, some researchers found that the body of the dynamic quadruped robot should not be set as a stiff beam because spine motion (intense spine curl and stretching) is usually apparent in the running of quadruped mammals (especially the cheetah). In order to make quadruped robot have better motion performance, now the quadruped robot with spine motion was studied gradually.

Biologists have conducted a lot of researches on spine motion of animals in the 1990s. Hildebrand [8] found that a primary function of spine motion was the kinematic 
extension of legs, which could effectively increase leg length. He also discussed how increased leg length due to spine motion increased running speed. Alexander et al. [9] presented that spine motion could supply extra power for high speed and the elastic structures in spine could improve system's energy efficiency. Schilling et al. [10] and Bertram et al. [11] studied the effect of spine motion on running gait selection and system stability. They found that spine motion enabled animals to choose a more rapid running gait while maintaining stability. These researches show that spine motion has many important and beneficial effects on animals' running performance, which makes robotic researchers believe that the quadruped robot with spine motion should be studied deeply.

Robotic researchers have also done some work on spine motion of quadruped robots. Çulha et al. [12] proposed a simplified sagittal plane model of quadruped mammals. In the body of the simplified model, there was a spinal driving joint. They investigated how quadrupedal bounding could be achieved in the presence of an actuated spinal joint and characterized associated performance improvements compared to bounding with a rigid robot body. Khoramshahi et al. [13] designed a quadruped robot with a spinal driving joint. Through experiments with the robot, they demonstrated that spine motion had the ability to reduce foot sliding on the ground and improve system's stability. Chen et al. [14] developed a planar quadruped robot with a spinal driving joint. The results of experiments with the robot and the simplified model indicate that spine motion can increase the average running speed and the intrinsic reason of speed increase is the improvement of the maximum horizontal thrust of rear leg. Cao et al. [15] researched the passive stability and feedback control for quadrupedal bounding with a segmented flexible spine. Hyun et al. [16] studied the gait control method for implementation of trotto-gallop transition and subsequent gallop on the MIT Cheetah (a quadruped robot with spine motion). Wang et al. [17] proposed a bio-inspired control strategy based on the motion nerve structure of animals for fast quadruped running with spine motion.

Although there have been some achievements in the studies of the quadruped robot with spine motion, there are still many shortcomings. All researches on the effect of spinal motion are carried out by doing experiments with either animals or robots. For robotic researchers, it's not enough to just know the effect of spine motion on system performance, because it has little help to develop a better quadruped robot. So far, the mechanism of spine motion has not been studied deeply, but it is helpful to set the parameters of mechanical structure and study control algorithm. In this paper, the mechanism of spine motion is studied deeply from dynamic view. Especially, the mechanism of spine motion about contact time is deeply discussed because contact time is a very important part of system performance. A planar simplified model of quadruped animals is set up. There are a spinal joint and a spinal spring in the body of the simplified model. Based on the Lagrange equation of energy conservation system, the dynamic equations of the simplified model for passive locomotion are derivated. A method that can generate passive periodic locomotion is proposed based on its dynamic equations and the Poincare mapping theory. According to the vertical spring oscillator model, the two-dimension planar locomotion of the simplified model is regarded as a special vibration in the vertical direction, its dynamic equations are rewritten, and the approximate formula of calculating contact time is obtained. Finally, the approximate formula of calculating contact time is verified by the simulation results of passive periodic locomotion, and the effect of spine motion on contact time is deeply discussed based on its dynamic equations, especially the approximate formula of calculating contact time.

\section{Model}

The cheetah is a very good biological blueprint for the quadruped robot with spine motion. In the running of the cheetah, the main movement occurs within the sagittal plane, and there are intense spine curl and stretching in the sagittal plane [18]. So, the simplified model of the cheetah that needs to be set up should be a two-dimension planar model.

Firstly, Some unimportant parts, like head, neck and tail, should be ignored directly. Secondly, according to Raibert's equivalent leg theory [2], the two front legs can be equivalent to a spring leg in front, and the two rear legs also can be equivalent to a spring leg in rear. Thirdly, the whole body can be divided into two parts and the two parts are connected by a spine joint and a spine spring. To sum up, the simplified model of the cheetah is shown in Figure 1, which is called the spine-joint model. The biggest difference between the spine-joint model set up in this paper and the simplified model proposed by Çulha et al. [12] is that the spine motion of the spine-joint model is flexible, which is more realistic and suitable for passive motion. Table 1 details the parameters of the spine-joint model. The parameters are evaluated by the average values of the adult cheetah [19] and that usually used in quadruped robots $[3,12,20,21]$.

\section{Dynamic Equations}

Passive locomotion is energy conservation locomotion under the given initial condition [22], which can rule out energy changing interference. So, this paper studies the mechanism of spine motion based on the passive 


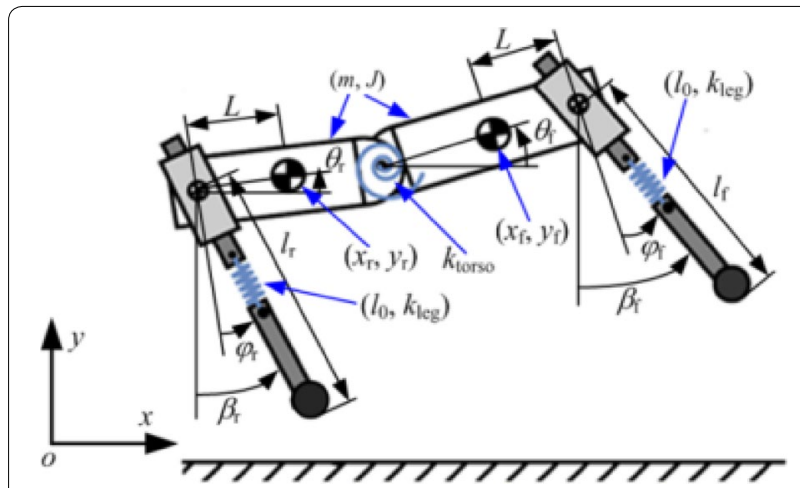

Figure 1 Simplified model of the cheetah

Table 1 Parameters of the spine-joint model

\begin{tabular}{ll}
\hline Parameter & Value \\
\hline Half body mass $m(\mathrm{~kg})$ & 25 \\
Half body length $L(\mathrm{~m})$ & 0.4 \\
Spine spring stiffness $k_{\text {torso }}(\mathrm{N} \cdot \mathrm{m} / \mathrm{rad})$ & 6 \\
Leg free length $I_{0}(\mathrm{~m})$ & 1.5 \\
Leg spring stiffness $k_{\text {leg }}(\mathrm{N} / \mathrm{m})$ & 18,000 \\
\hline
\end{tabular}

periodic locomotion of the spine-joint model. In order to get passive locomotion, the following assumptions are given for the spine-joint model:

1. All movements do not consume energy.

2. All joints are frictionless.

3. When leg collides with the ground, there is no energy loss, and the contact point can be regarded as a joint without friction.

Under these assumptions, the dynamic equations of the spine-joint model can be derived in the Cartesian coordinates shown in Figure 1, based on the Euler-Lagrange equation of energy conservation system. In the dynamic equations, the state vector $\boldsymbol{x}$ is:

$$
\boldsymbol{x}=\left[x, y, \theta_{\mathrm{f}}, \theta_{\mathrm{r}}, \dot{x}, \dot{y}, \dot{\theta}_{\mathrm{f}}, \dot{\theta}_{\mathrm{r}}\right]^{\mathrm{T}},
$$

where $(x, y)$ is the position of the body's center mass, $\theta_{\mathrm{f}}$ is the front half body's pitch angle around the horizontal axis, and $\theta_{\mathrm{r}}$ is the rear half body's pitch angle around the horizontal axis.

According to the bounding gait [23], the passive periodic locomotion of the spine-joint model can be divided into four phases (front leg stance phase, gathered flight phase, rear leg stance phase, and extended flight phase), and the four phases are converted through four triggering events (front leg liftoff event, rear leg touchdown event, rear leg liftoff event, and front leg touchdown event), as shown in Figure 2. Because constraints are different in the four phases, the dynamic equations of each phase should be derived separately, and the mathematical expression of each triggering event should also be got.

\section{Passive Periodic Locomotion}

According to dynamic equations, how to obtain the passive periodic locomotion of the spine-joint model? The answer is to set the initial value of each variable properly. Because there are ten variables in dynamic equations, eight state variables and two related variables (the front leg's landing angle around the vertical axis $\beta_{\mathrm{f}}^{\text {td }}$ and the rear leg's landing angle around the vertical axis $\beta_{\mathrm{r}}^{\text {td }}$ ), a method that can automatically search for appropriate variables should be proposed.

Dynamic equations show the passive locomotion of the spine-joint model is a highly nonlinear continuous system, which is not good for determining whether the final solution trajectory is cyclical. So using the Poincare map theory, this continuous system can be mapped to a discrete system $\boldsymbol{P}$ and searching for appropriate variables is to solve fixed points of the discrete system $\boldsymbol{P}$. The highest point of the trajectory is selected as the Poincare map point. In this point, the state variable $\dot{y}$ is zero, which has no effect on whether the trajectory is cyclical or not, and the two related variables $\left(\beta_{\mathrm{f}}^{\text {td }}\right.$ and $\left.\beta_{\mathrm{r}}^{\text {td }}\right)$ can be included directly. Such, the equation for solving the Poincare map fixed point of the discrete system $\boldsymbol{P}$ is:
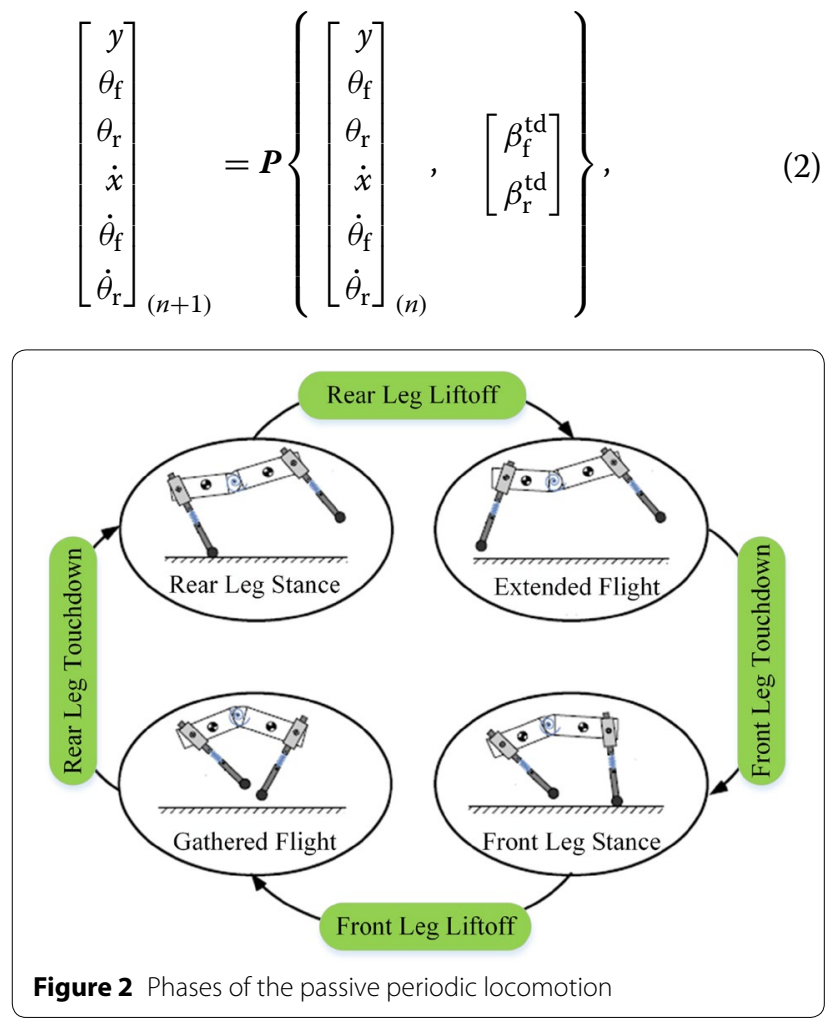
The Newton-Raphson algorithm is a very efficient method for solving Eq. (2). Under the estimated initial values of each variable, a fixed point of the discrete system $\boldsymbol{P}$ can be searched automatically, based on the dynamic equations and the Newton-Raphson algorithm. So, the passive periodic locomotion of the spine-joint model can be obtained by using the fixed point in Poincare map as the initial values of each variable. For example, when the initial state vector $\boldsymbol{x}$ is $[0 \mathrm{~m}, 1.55 \mathrm{~m},-0.3 \mathrm{rad}, 0.3 \mathrm{rad}, 10 \mathrm{~m} / \mathrm{s}$, $0 \mathrm{~m} / \mathrm{s}, 7 \mathrm{rad} / \mathrm{s}, 7 \mathrm{rad} / \mathrm{s}]^{\mathrm{T}}, \beta_{\mathrm{f}}^{\text {td }}$ and $\beta_{\mathrm{r}}^{\text {td }}$ are both $0.62 \mathrm{rad}$, the passive locomotion is periodic, the trajectories of each state variable in one cycle are shown in Figure 3.

\section{Effect of Spine Motion on Contact Time}

Contact time refers to the duration of leg from landing the ground to flight. During this period, leg collides with the ground, producing force to support body locomotion [24]. So the length of contact time is directly related to locomotion performance.

In the passive periodic locomotion of the spine-joint model, the front leg's contact time and the hind leg's contact time are equal, which is shown in Figure 3. So the following of this paper only studies the hind leg's contact time. In order to study the effect of spine motion on contact time, a comparison model which has not spine motion should be established. Through removing the spine spring and locking the spine joint, the comparison model with the spine-joint model can be got, which is the spring-beam model $[4,6,25]$.

\subsection{Simulation Results}

Contact time can be got according to the passive periodic locomotion of the spine-joint model and the spring-beam model. Figure 4 is the trajectories of contact time along with horizontal speed. The contact time of the spinejoint model and the spring-beam model both decreases with the increase of horizontal speed, shown in Figure 4, which is consistent with the conclusions of Refs. [24, 26]. Ref. [26] also demonstrates horizontal speed exerts effect on contact time by means of the centrifugal force which can enhance system's natural frequency.

Figure 4 also shows that the contact time of the spinejoint model is slightly greater than that of the spring-beam model at the same horizontal speed. When horizontal speed is $7 \mathrm{~m} / \mathrm{s}$, the contact time of the spine-joint model is $0.1596 \mathrm{~s}$, and the contact time of the spring-beam model is $0.1427 \mathrm{~s}$. when horizontal speed is $10 \mathrm{~m} / \mathrm{s}$, the contact time of the spine-joint model is $0.1380 \mathrm{~s}$, and the contact time of the spring-beam model is $0.1274 \mathrm{~s}$. That suggests the spine motion has little effect on contact time.

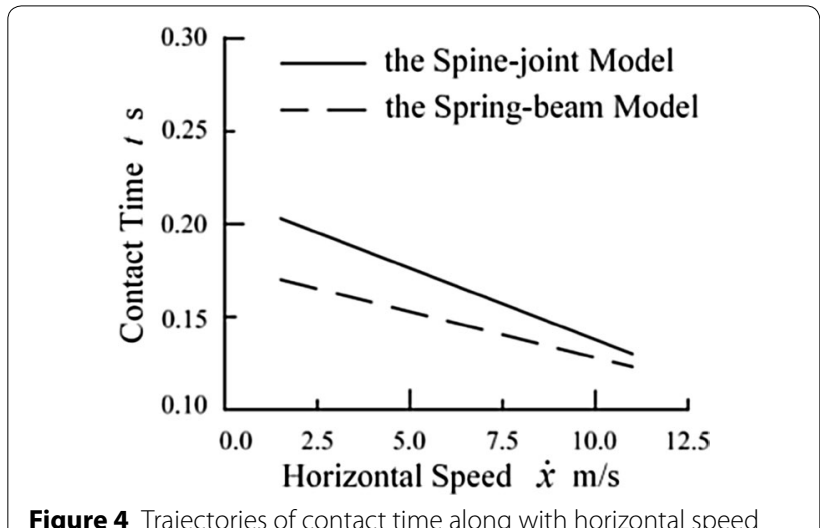

Figure 4 Trajectories of contact time along with horizontal speed

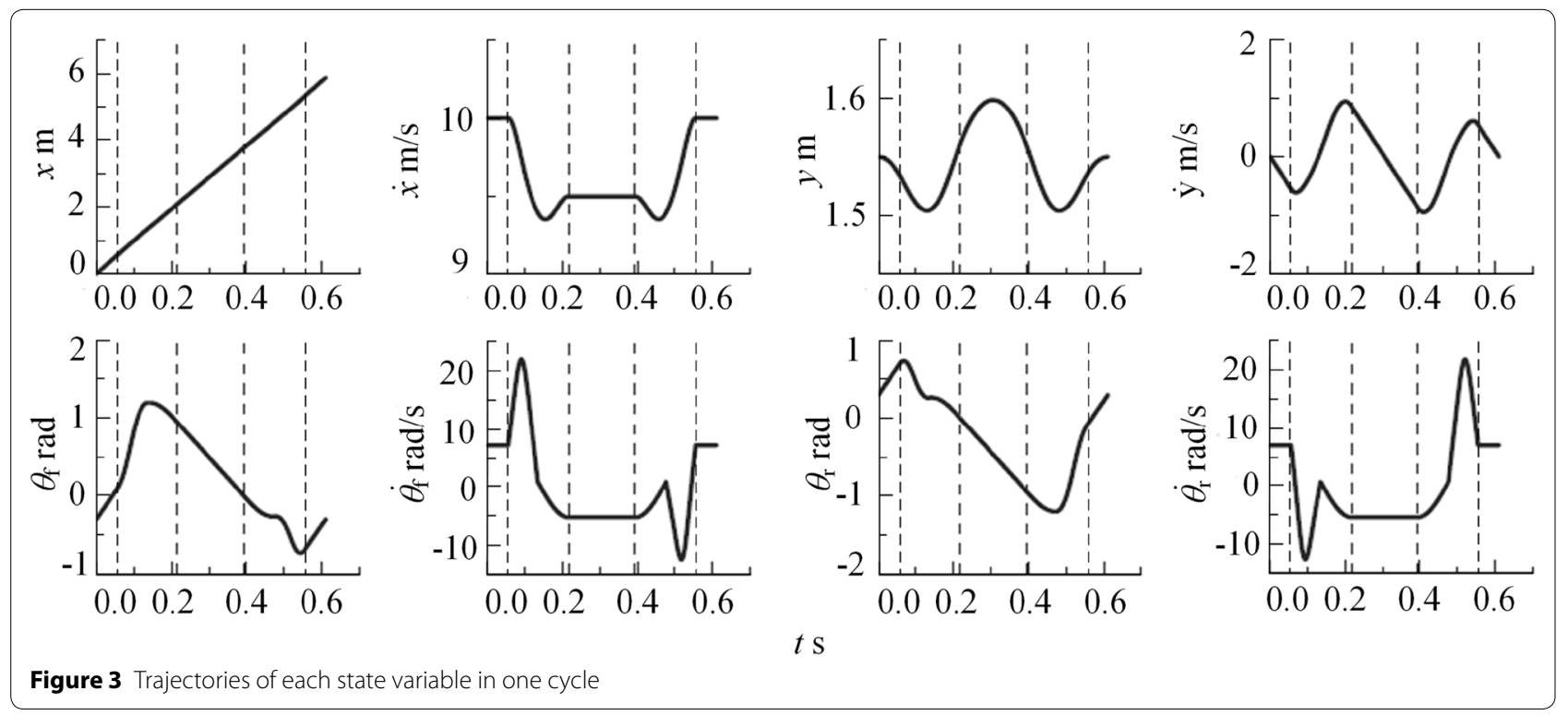




\subsection{Mechanism Analysis}

In Ref. [26], a method of studying the contact time of the spring-beam model is proposed, which is based on system's dynamics equations and regards the spring-beam model's two-dimension planar locomotion as a special vibration in the vertical direction. As this method is feasible to the spring-mass model $[5,27]$ and the springbeam model of quadruped robot, this paper extends it to the spine-joint model.

The vertical spring oscillator model is a basic model for studying vibration, its dynamic equations in Polar coordinates and its contact time calculation formula can be got in Ref. [26]. According to the change of Cartesian coordinates to Polar coordinates, the spine-joint model's dynamic equations in Polar coordinates can be derived. The two-dimensional locomotion of the spinejoint model is also regarded as a special vibration in the vertical direction, and its dynamic equations in rear leg stance phase are rewritten again based on the form of the vertical spring oscillator model's dynamic equations. So, according to the contact time calculation formula of the vertical spring oscillator model, the contact time approximate calculation formula of the spine-joint model can be derived, as shown in Eq. (3): $l_{\mathrm{r}}$ is the rear leg's real-time length, and $\beta_{\mathrm{r}}$ is the rear leg's real-time contact angle around the vertical axis.

Figure 5 shows the calculation results for the contact time of the spine-joint model according to Eq. (3). Comparing the calculation results with the simulation results, the two values are almost equal. When horizontal speed is $7 \mathrm{~m} / \mathrm{s}$, the simulation value is $0.1596 \mathrm{~s}$, and the calculation value is $0.1567 \mathrm{~s}$. When horizontal speed is $10 \mathrm{~m} / \mathrm{s}$, the simulation value is $0.1380 \mathrm{~s}$, and the calculation value is $0.1329 \mathrm{~s}$. This means that the approximate calculation formula for the contact time of the spine-joint model is correct and the method, regarding the two-dimensional locomotion as a special vibration in the vertical direction, is also effective for the spine-joint model.

Equation (3) shows that $B K$ can enhance system's vibration angular frequency $\omega$. Figure $6(\mathrm{a})$ and (b) are the calculation results of $B K$ for the spine-joint model and its comparison model, the spring-beam model, respectively. The calculation formula of $B K$ for the spring-beam model can be got from Ref. [26]. In Figure 6, compared with other leg stiffness enhancement coefficients, $B K_{\mathrm{c}}$ of both the spine-joint model and the spring-beam model has a significant increase with the

$$
\begin{aligned}
& t_{\text {stance }}=\frac{\pi}{\omega}, \\
& \omega=\sqrt{\left(1+B K_{\mathrm{c}}+B K_{\mathrm{g}}+B K_{\alpha}+B K_{\theta \mathrm{h}}+B K_{\omega \theta \mathrm{f}}+B K_{\omega \theta \mathrm{r}}\right)} \sqrt{\frac{k_{\mathrm{leg}}}{2 m}}, \\
& B K_{\mathrm{c}}=\frac{\int_{t^{\mathrm{td}}}^{t^{\mathrm{lf}}} A_{\mathrm{c}} \mathrm{d} t}{\int_{t^{\mathrm{dd}}}^{t^{\mathrm{f}}} A_{\mathrm{k}} \mathrm{d} t}, B K_{\mathrm{g}}=\frac{\int_{t^{\mathrm{dd}}}^{t^{\mathrm{lf}}} A_{\mathrm{g}} \mathrm{d} t}{\int_{t^{\mathrm{dd}}}^{t^{\mathrm{lf}}} A_{\mathrm{k}} \mathrm{d} t}, B K_{\alpha}=\frac{\int_{t^{\mathrm{td}}}^{t^{\mathrm{ff}}} A_{\alpha} \mathrm{d} t}{\int_{t^{\mathrm{td}}}^{t^{\mathrm{f}}} A_{\mathrm{k}} \mathrm{d}}, \\
& B K_{\theta \mathrm{h}}=\frac{\int_{t^{\mathrm{dd}}}^{t^{\mathrm{lf}}} A_{\theta \mathrm{h}} \mathrm{d} t}{\int_{t^{\mathrm{td}}}^{t^{\mathrm{f}}} A_{\mathrm{k}} \mathrm{d} t}, B K_{\omega \theta \mathrm{f}}=\frac{\int_{t^{\mathrm{td}}}^{t^{\mathrm{ff}}} A_{\omega \theta \mathrm{f}} \mathrm{d} t}{\int_{t^{\mathrm{td}}}^{t^{\mathrm{f}}} A_{\mathrm{k}} \mathrm{d} t}, B K_{\omega \theta \mathrm{r}}=\frac{\int_{t^{\mathrm{td}}}^{t^{\mathrm{lf}}} A_{\omega \theta \mathrm{r}} \mathrm{d} t}{\int_{t^{\mathrm{td}}}^{t^{\mathrm{f}}} A_{\mathrm{k}} \mathrm{d} t} \\
& A_{\mathrm{c}}=l_{\mathrm{r}} \dot{\beta}_{\mathrm{r}}^{2}, A_{\mathrm{k}}=\frac{k_{\mathrm{leg}}}{2 m}\left(l_{0}-l_{\mathrm{r}}\right), A_{\mathrm{g}}=-\cos \left(\beta_{\mathrm{r}}\right) g, \\
& A_{\alpha}=\left(-\frac{L}{2} \cos \left(\theta_{\mathrm{f}}-\beta_{\mathrm{r}}\right)-\frac{3 L}{2} \cos \left(\theta_{\mathrm{r}}-\beta_{\mathrm{r}}\right)\right) \frac{\ddot{\alpha}}{2}, \\
& A_{\theta \mathrm{h}}=\left(-\frac{L}{2} \cos \left(\theta_{\mathrm{f}}-\beta_{\mathrm{r}}\right)-\frac{3 L}{2} \cos \left(\theta_{\mathrm{r}}-\beta_{\mathrm{r}}\right)\right) \ddot{\theta}_{\mathrm{h}}, \\
& A_{\omega \theta \mathrm{f}}=\frac{L}{2} \sin \left(\theta_{\mathrm{f}}-\beta_{\mathrm{r}}\right) \dot{\theta}_{\mathrm{f}}^{2}, A_{\omega \theta \mathrm{r}}=\frac{3 L}{2} \sin \left(\theta_{\mathrm{r}}-\beta_{\mathrm{r}}\right) \dot{\theta}_{\mathrm{r}}^{2}, \\
& \alpha=\theta_{\mathrm{f}}-\theta_{\mathrm{r}}, \quad \theta_{\mathrm{h}}=\frac{\theta_{\mathrm{f}}+\theta_{\mathrm{r}}}{2},
\end{aligned}
$$

where $\omega$ is the system's vibration angular frequency, $B K$, a dimensionless quantity, is called the enhancement coefficient of leg stiffness, $A$, a combinatorial dimension quantity, is called the enhancement coefficient of leg length, increase of horizontal speed. So, the way of horizontal speed exerting effect on the contact time of the spinejoint model is also centrifugal force, which can be got from the calculation formula of $B K_{\mathrm{c}}$. 
Why is the contact time of the spine-joint model slightly longer than that of the spring-beam mode? According to the contact time approximate calculation formula of the spine-joint model and the spring-beam model, Figure 6 also shows that the contact time of the

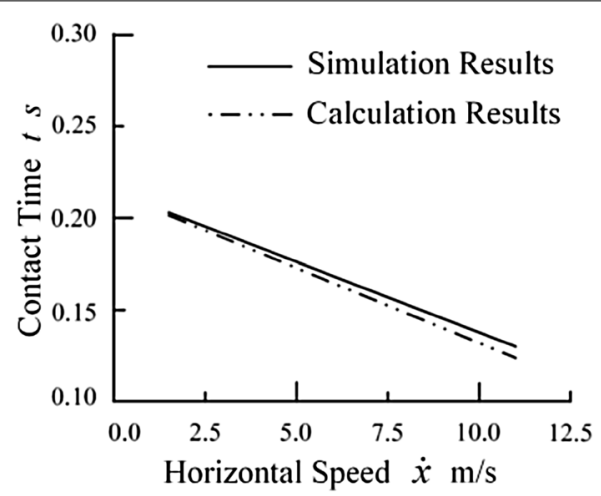

Figure 5 Calculation results of contact time
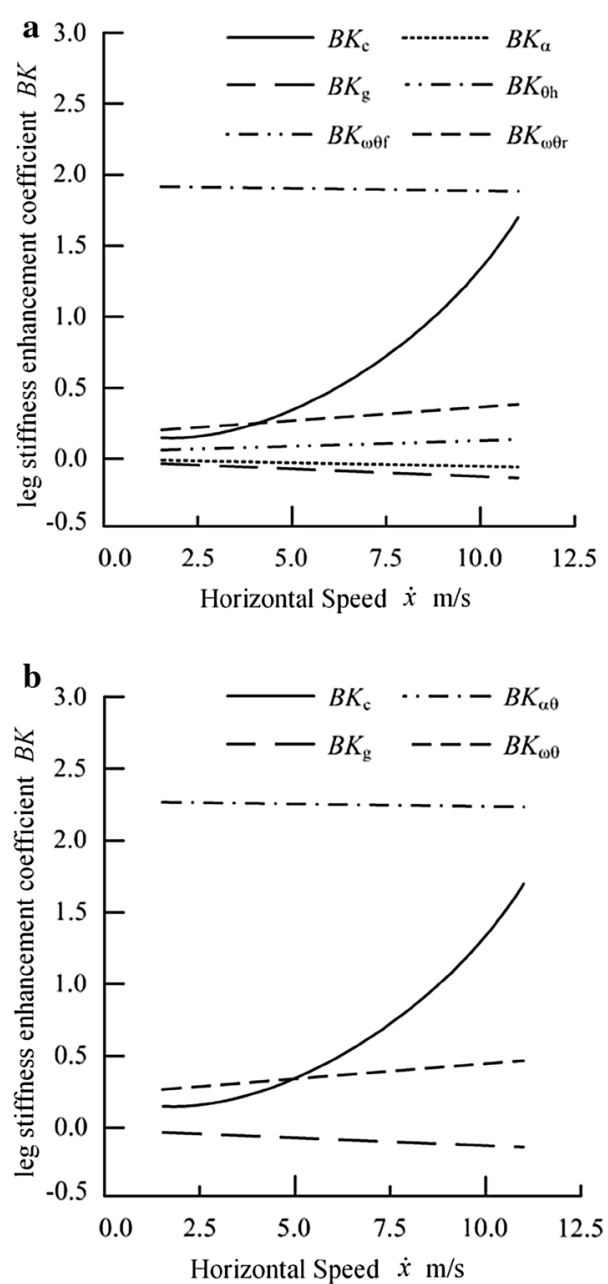

Figure 6 Calculation results of leg stiffness enhancement coefficients: a spine-joint model and $\mathbf{b}$ spring-beam model

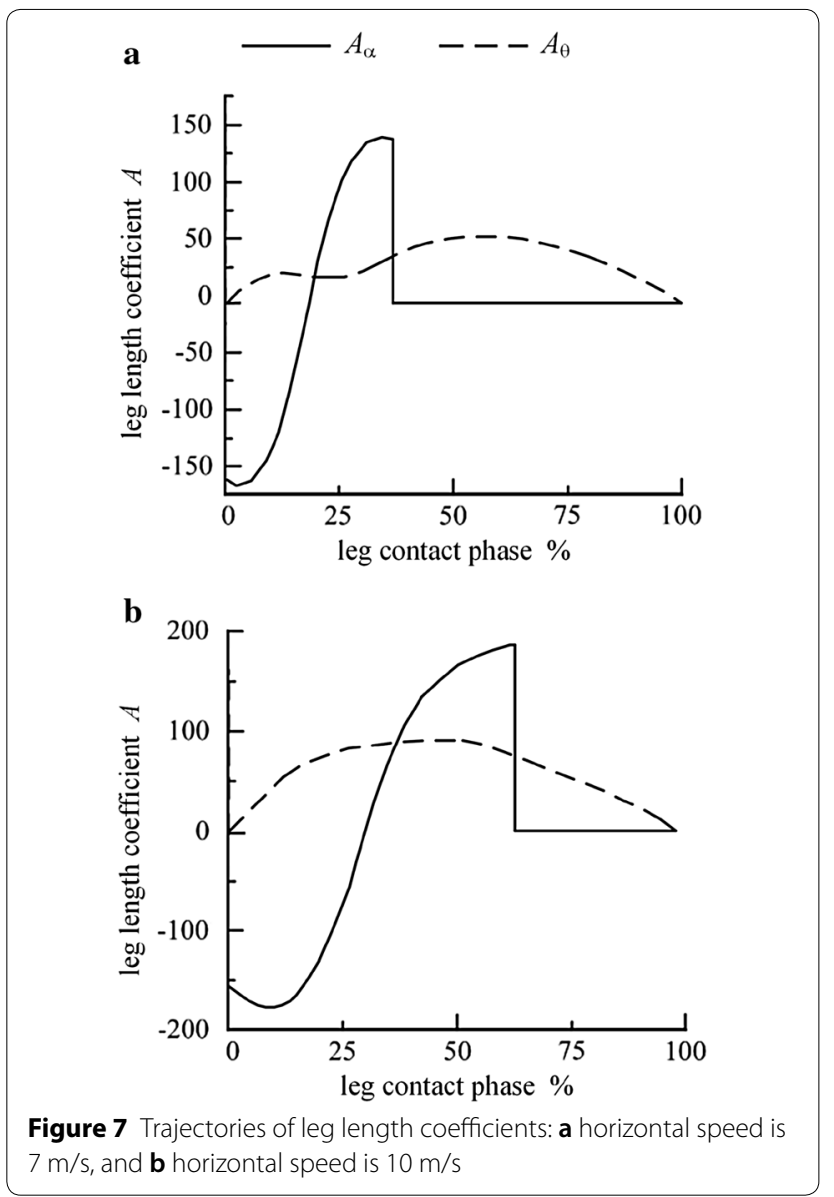

spine-joint model is almost determined by the values of $B K_{\mathrm{c}}$ and $B K_{\theta \mathrm{h}}$, and for the spring-beam model it is the values of $B K_{\mathrm{c}}$ and $B K_{\alpha \theta}$. At the same horizontal speed, the $B K_{\mathrm{c}}$ of the spine-joint model is almost equal to that of the spring-beam model, and $B K_{\theta \mathrm{h}}$ is slightly less than $B K_{\alpha \theta}$. When horizontal speed is $7 \mathrm{~m} / \mathrm{s}$, the $B K_{\mathrm{c}}$ and $B K_{\theta \mathrm{h}}$ of the spine-joint model are 0.6165 and 1.8996 , and the $B K_{\mathrm{c}}$ and $B K_{\alpha \theta}$ of the spring-beam model are 0.6147 and 2.2397. When horizontal velocity is $10 \mathrm{~m} / \mathrm{s}$, the $B K_{\mathrm{c}}$ and $B K_{\theta \mathrm{h}}$ of the spine-joint model are 1.3331 and 1.8834, and the $B K_{\mathrm{c}}$ and $B K_{\alpha \theta}$ of the spring-beam model are 1.3354 and 2.2131. Based on the calculation formulas of $B K_{\theta \mathrm{h}}$ and $B K_{\alpha \theta}, B K_{\theta \mathrm{h}}$ and $B K_{\alpha \theta}$ are both the leg stiffness enhancement coefficients produced by the inertia force of body pitching. So spine motion indeed has little effect on contact time, but it can slightly reduce body pitching which is helpful to keep stability based on the research conclusions of Refs. [22, 26, 28].

The calculation formula of $B K_{\alpha}$ in Eq. (3) shows that $B K_{\alpha}$ is the leg stiffness enhancement coefficient produced directly by spine motion. But Figure 6 shows $B K_{\alpha}$ is almost zero, which also testifies spine motion indeed has 
little effect on contact time. Why is $B K_{\alpha}$ almost zero? The calculation formula of $B K_{\alpha}$ shows that the value of $B K_{\alpha}$ is determined by $A_{\alpha}$. Figure 7 is the trajectories of $A_{\alpha}$ and $A_{\theta \mathrm{h}}$ in the spine-joint model's rear leg contact phase. Figure 7 (a) is calculated when horizontal speed is $7 \mathrm{~m} / \mathrm{s}$, and Figure 7(b) is calculated when horizontal speed is $10 \mathrm{~m} / \mathrm{s}$. Both Figure 7(a) and (b) show that the trajectory of $A_{\alpha}$ presents an approximate odd symmetry in the whole rear leg contact phase, which is just the reason why $B K_{\alpha}$ is almost zero. Although spine motion has little effect on contact time, Figure 7 also shows that spine motion can regulate leg stiffness in the leg contact phase, making it softer first and stiffer later, which is helpful to optimize leg force and improve energy efficiency based on the research conclusions of Refs. [19, 29, 30].

\section{Conclusions}

(1) The spine-joint model set up in this paper has a spine joint and a spine spring, which is a suitable simplified model for the cheetah. Based on the dynamic equations of the spine-joint model and the Poincare map, the passive periodic locomotion is obtained. The approximate calculation formula of contact time derived in this paper demonstrates that the studying method, regarding the two-dimensional locomotion as a special vibration in the vertical direction, is also viable for the spinejoint model.

(2) For the spine-joint model, contact time also decreases with the increase of horizontal speed, and the reason is also the centrifugal force, which is the same as the spring-beam model and the springmass model.

(3) Spine motion indeed has little effect on contact time, which is testified by the passive periodic locomotion of the spine-joint model and the approximate calculation formula of contact time. And the study results also show spine motion can slightly reduce body pitching and regulate leg stiffness in leg contact phases, making it softer first and stiffer later, which is helpful to system's other performance, such as stability, leg force, and so on.

\section{Authors' Contributions}

YB was in charge of the whole trial; QL wrote the manuscript; WY and JZ assisted with mechanism analysis; $\mathrm{CL}$ assisted with simulation. All authors read and approved the final manuscript.

\section{Author Details}

${ }^{1}$ Institute of Chemical Materials, China Academy of Engineering Physics, Mianyang 621999, China. ${ }^{2}$ College of Automation, Harbin Engineering University, Harbin 150000, China.

\section{Authors' Information}

Qi Liu, born in 1988, is currently an engineer at Institute of Chemical Materials, China Academy of Engineering Physics, China. He received his bachelor degree from Harbin Engineering University, China, in 2015. His research interest is bionic robot.

Yannian Bao, born in 1975, is currently the deputy director of the special equipment center at Institute of Chemical Materials, China Academy of Engineering Physics, China. He received his bachelor degree from Chongqing University, China, in 2003. His research interest is robotic technology and its application.

Wei Yu, born in 1989, is currently an engineer at Institute of Chemical Materials, China Academy of Engineering Physics, China. He received his bachelor degree from Harbin Engineering University, China, in 2015.

Jianming Zhang, born in 1983, is currently an engineer at Institute of Chemical Materials, China Academy of Engineering Physics, China. He received his bachelor degree from Sichuan University, China, in 2011.

Chao Li, born in 1991, is currently a PhD candidate at College of Automation, Harbin Engineering University, China. He received his bachelor degree from Harbin Engineering University, China, in 2015. His research interest is flexible robot.

Xinru Xie, born in 1992, is currently a PhD candidate at College of Automation, Harbin Engineering University, China. He received his bachelor degree from Harbin Engineering University, China, in 2016. His research interest is flexible robot

\section{Acknowledgements}

The authors sincerely thanks to Professor Yude Fan of Institute of Chemical Materials, China Academy of Engineering Physics and Professor Qidan Zhu of College of automation, Harbin engineering university for their critical discussion and reading during manuscript preparation.

\section{Competing Interests}

The authors declare that they have no competing interests.

\section{Funding}

Supported by National Natural Science Foundation of China and China Academy of Engineering Physics (NSAF, Grant No. U1530119).

Received: 15 July 2017 Accepted: 20 February 2019

Published online: 07 March 2019

\section{References}

[1] R S Mosher. Test and evaluation of a versatile walking truck. Proc. Off-Road Mobility Research Symp., 1968: 359-379.

[2] M H Raibert. Legged robots that balance. Cambridge MA: The MIT Press, 1986.

[3] M H Raibert, K Blankespoor, G Nelson, et al. Bigdog, the rough-terrain quadruped robot. IFAC Proceedings Volumes, 2008, 41(2): 10822-10825.

[4] A Spröwitz, A Tuleu, M Vespignani, et al. Towards dynamic trot gait locomotion: design, control, and experiments with Cheetah-cub, a compliant quadruped robot. The International Journal of Robotics Research, 2013, 32(8): 932-950.

[5] B Miller, J Schmitt, J E Clark. Quantifying disturbance rejection of SLIP-like running systems. The International Journal of Robotics Research, 2012, 31(5): 573-587.

[6] L Ding, H B Gao, Z Q Deng, et al. Foot-terrain interaction mechanics for legged robots: Modeling and experimental validation. The International Journal of Robotics Research, 2013, 32(13): 1585-1606.

[7] M Kalakrishnan, J Buchli, P Pastor, et al. Learning, planning, and control for quadruped locomotion over challenging terrain. The International Journal of Robotics Research, 2011, 30(2): 236-258.

[8] M Hildebrand. Motions of the running cheetah and horse. Journal of Mammalogy, 1959, 40(4): 481-495.

[9] R M N Alexander, N J Dimery, R F Ker. Elastic structures in the back and their role in galloping in some mammals. Journal of Zoology, 1985, 207(4): 467-482.

[10] N Schilling, R Hackert. Sagittal spine movements of small therian mammals during asymmetrical gaits. Journal of Experimental Biology, 2006, 209(19): 3925-3939. 
[11] J E A Bertram, A Gutmann. Motions of the running horse and cheetah revisited: fundamental mechanics of the transverse and rotary gallop. Journal of the Royal Society Interface, 2009, 6(35): 549-559.

[12] U Çulha, U Saranli. Quadrupedal bounding with an actuated spinal joint. IEEE International Conference on Robotics and Automation, Shanghai, China, May 9-13, 2011: 1392-1397.

[13] M Khoramshahi, A Sprowitz, A Tuleu, et al. Benefits of an active spine supported bounding locomotion with a small compliant quadruped robot. IEEE International Conference on Robotics and Automation, Karlsruhe, Germany, May 6-10, 2013: 3329-3334.

[14] D L Chen, Q liu, L T Dong, et al. Effect of spine motion on mobility in quadruped running. Chinese Journal of Mechanical Engineering, 2014, 27(6): 1150-1156.

[15] Q Cao, I Poulakakis. Quadrupedal bounding with a segmented flexible torso: passive stability and feedback control. Bioinspiration \& Biomimetics, 2013, 8(4): 046007.

[16] D J Hyun, J Lee, S I Park, et al. Implementation of trot-to-gallop transition and subsequent gallop on the MIT Cheetah I. The International Journal of Robotics Research, 2016, 35(13): 1627-1650.

[17] C Wang, T Zhang, X Wei, et al. Bio-inspired control strategy study for the quadruped robot with a segmented spine. Industrial Robot: An International Journal, 2017, 44(1): 85-93.

[18] M Hildebrand. Further studies on locomotion of the cheetah. Journal of Mammalogy, 1961, 42(1): 84-91.

[19] P E Hudson, S A Corr, A M Wilson. High speed galloping in the cheetah (Acinonyx jubatus) and the racing greyhound (Canis familiaris): spatiotemporal and kinetic characteristics. Journal of Experimental Biology, 2012, 215(14): 2425-2434.

[20] K Karakasiliotis, R Thandiackal, K Melo, et al. From cineradiography to biorobots: an approach for designing robots to emulate and study animal locomotion. Journal of the Royal Society Interface, 2016, 13(119): 20151089.
[21] Q Zhao, B Ellenberger, H Sumioka, et al. The effect of spine actuation and stiffness on a pneumatically-driven quadruped robot for cheetah-like locomotion. IEEE International Conference on Robotics and Biomimetics (ROBIO), 2013: 1807-1812.

[22] C D Remy, K Buffintion, R Siegwart. Stability analysis of passive dynamic walking of quadrupeds. The International Journal of Robotics Research, 2010, 29(9): 1173-1185

[23] L T Phan, Y H Lee, Y H Lee, et al. Study on quadruped bounding with a passive compliant spine. IEEE International Conference on Intelligent Robots and Systems (IROS), 2017: 2409-2414.

[24] D F Hoyt, S J Wickler, E A Cogger. Time of contact and step length: the effect of limb length, running speed, load carrying and incline. Journal of Experimental Biology, 2000, 203(2): 221-227.

[25] I Poulakakis, J A Smith, M Buehl. Modeling and experiments of untethered quadrupedal running with a bounding gait: The Scout II robot. The International Journal of Robotics Research, 2005, 24(4): 239-256.

[26] Q Deng, S G Wang, Q H Liang, et al. The effect of body pitching on leg-spring behavior in quadruped running. Journal of Bionic Engineering, 2010, 7(3): 219-227

[27] H R Vejdani, A Wu, H Geyer, et al. Touch-down angle control for springmass walking. IEEE International Conference on Robotics and Automation (ICRA), 2015: 5101-5106.

[28] D L Chen, N Li, G Liu, et al. Effects of spine motion on foot slip in quadruped bounding. Applied Bionics and Biomechanics, 2018: 8097371.

[29] L Righetti, J Buchli, M Mistry, et al. Optimal distribution of contact forces with inverse-dynamics control. The International Journal of Robotics Research, 2013, 32(3): 280-298.

[30] S Seok, A Wang, M Y M Chuah, et al. Design principles for energy-efficient legged locomotion and implementation on the MIT cheetah robot. Transactions on Mechatronics, 2015, 20(3): 1117-1129.

\section{Submit your manuscript to a SpringerOpen ${ }^{\odot}$ journal and benefit from:}

- Convenient online submission

- Rigorous peer review

- Open access: articles freely available online

- High visibility within the field

- Retaining the copyright to your article

Submit your next manuscript at $\boldsymbol{\nabla}$ springeropen.com 